\title{
Design, Optimization and Calibration of an HFB-based ADC
}

\author{
A. LESELLIER, O. JAMIN \\ NXP Semiconductors \\ Caen, France \\ amandine.lesellier@nxp.com
}

\author{
J.-F. BERCHER*, O. VENARD ${ }^{+}$ \\ ESIEE-Université de Paris-Est \\ Noisy-le-Grand, France \\ * LIGM, ${ }^{+}$LaMIPS
}

\begin{abstract}
We describe the design of an HFB-based ADC targeted towards the digitization of a very large band for Software Defined Radio applications. We present an original procedure for the optimization of the synthesis filters, when the front-end analysis filters use standard low-cost analog filters. We also address the calibration of the device, namely the identification of the actual analog filters, and highlight the impact of the identification and of measurement errors on the overall performances.
\end{abstract}

\section{INTRODUCTION}

For many applications such as Software Defined Radio (SDR), the interesting information could be located in many subbands, anywhere in a much larger band. An attractive way to cope with this problem is to digitize the whole band, and then, to select the information in digital domain. This flexibility has a cost and requires a high-performance Analogto-Digital Converter (ADC), which is the bottleneck of the reception chain. Indeed, such an ADC must be able to convert a very large band, at a very high sampling rate and with a very good resolution.

The current Analog-to-Digital Converters (ADC) architectures are not adapted to such an application. Flash ADCs, pipeline ADCs, Successive Approximation Register (SAR) ADCs and $\Sigma \Delta$ ADCs are either high speed or high resolution. According to the literature, parallel structures for ADCs are a key for the design of high-speed, high-resolution data converters. Time-interleaving (TI) [1], Parallel DeltaSigma $(\pi \Delta \Sigma)$ ADC [2], Hybrid Filter Banks (HFB) [3] are potential architectures.

An analysis of an HFB-based ADC solution is proposed in this paper, together with original contributions, namely concerning (a) the design of the digital synthesis bank, and (b) the initial calibration of the device. These are valid whatever the type of ADC. In the present study, quantization errors have not been considered. The principle of the HFB and main relations are recalled in section II. In the next section, we explain the main implementation choices, present an algorithm for the optimization of synthesis filters and then, in section IV we focus on the issue of identification of the analog analysis filters, that is to say, the way to have access to the actual transfer functions of the analog filters. The results obtained by simulation with Matlab are presented and discussed in section V.

\section{PRINCIPLE OF HFB}

Hybrid filter banks (HFB) employ both a bank of analog filters $\mathrm{H}_{\mathrm{i}}$, called analysis bank, and a bank of digital filters $\mathrm{F}_{\mathrm{i}}$, called synthesis bank. The structure of such an HFB-based ADC is shown in Fig. 1.

The analog filters split the input into subbands. Each ADC then digitizes each subband at the sampling rate of $F_{S}=F e / M$, where $M$ is the number of channels and $F e$, the sampling rate of the global ADC. The analysis structure is then followed by upsamplers by $M$ and digital synthesis filters. Finally, the $M$ channels are recombined at the output, which completes the architecture. So doing, the output of the HFB is the digital equivalent of the analog input, with more or less reconstruction errors.

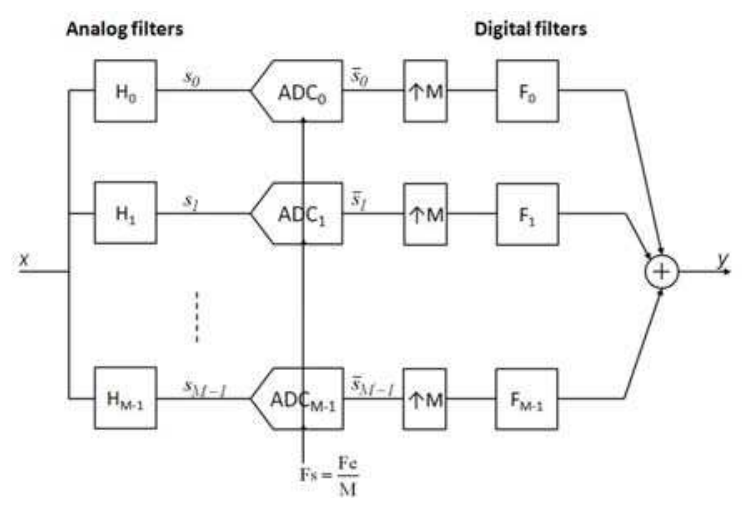

Figure 1. HFB architecture

Actually, the HFB shall be designed so as to fulfill, as far as possible, the so-called Quasi-Perfect Reconstruction (QPR) condition. This leads to the main advantage of the architecture which is that aliasing is tolerated in each subband, because it is attenuated, ideally suppressed, by the recombination of the outputs of the synthesis filters. The following equations only stand for a two-channel HFB-based ADC, as studied in [4]. Denoting $X(j \omega)$ and $Y\left(e^{j \omega T e}\right)$ the Fourier transforms of the input $\mathrm{x}(\mathrm{t})$ and the output $\mathrm{y}(\mathrm{n})$ of the system, we have: 


$$
\begin{gathered}
Y\left(e^{j \omega T e}\right)=G_{0}(j \omega) \cdot X(j \omega)+G_{1}(j \omega) \cdot X\left(j \omega-j \frac{\pi}{T e}\right) \\
\text { with } \quad G_{0}(j \omega)=H_{0}(j \omega) \cdot F_{0}\left(e^{j \omega T e}\right)+H_{1}(j \omega) \cdot F_{1}\left(e^{j \omega T e}\right)(1)
\end{gathered}
$$

and

$$
G_{1}(j \omega)=H_{0}\left(j \omega-j \frac{\pi}{T e}\right) \cdot F_{0}\left(e^{j \omega T e}\right)+H_{1}\left(j \omega-j \frac{\pi}{T e}\right) \cdot F_{1}\left(e^{j \omega T e}\right)(2)
$$

In these relations, $\mathrm{G}_{0}$ is the distortion (or transfer) function and $G_{1}$ is called the aliasing function. The overall goal of the design of the HFB is to approach a perfect reconstruction (PR). In order to obtain perfect reconstruction, $\mathrm{G}_{0}$ should be a pure delay and $G_{1}$ should be null. These conditions could be reached with a digital bank but it seems that $\mathrm{PR}$ is not possible with Hybrid Filter Banks.

We have selected here a two-channel HFB-based ADC in order to avoid implementation of analog bandpass filters that have a cost in terms of surface and complexity. Then, $\mathrm{H}_{0}$ is an analog lowpass filter, and $\mathrm{H}_{1}$ an analog highpass filter. Regarding the digital filters, $F_{0}$ is a lowpass filter and $F_{1}$ a highpass filter. As already mentioned, PR is not achievable with HFB, so we have determined a specification of maximum distortion of the transfer function (1), called $\mathrm{G}_{0} \mathrm{dBmax}$, and maximum of aliasing rejection (2), called $\mathrm{G}_{1} \mathrm{dBmax}$, so that the digitized output is good enough for our digital processing. We target: $\mathrm{G}_{0} \mathrm{dBmax}<0.5 \mathrm{~dB}$ and $\mathrm{G}_{1} \mathrm{dBmax}<-70 \mathrm{~dB}$.

So as to improve the performances, it has been shown in [5] that a guard band can be introduced, i.e. the global sampling rate is chosen greater than the Nyquist frequency. In this case, $G_{0}$ and $G_{1}$ are defined on the band of interest.

There are many ways to implement an HFB-based ADC. These are discussed below, with highlights on our particular choices.

\section{IMPLEMENTATION}

\section{A. Choice of the filters}

In this architecture, the choice of the filter banks is very important. Many solutions have been proposed to construct an HFB-based ADC. As PR is reachable with digital filter banks, one of them consists in using a Z-to-S transform to create the continuous-time analog filters [6]. The major drawback with this approach is that the order of each analog filter equals the order of the prototype multiplied by the degree of the transform. Hence this approach results in a high order analysis filter bank [7]. A second approach consists in adjusting the poles and zeros of the analog filters so as to minimize the reconstruction errors. Another solution is to first optimize the analog filters and then, with the analog filters fixed, design the digital synthesis filters [8]. In [9], it is proposed to use power complementary filters for a two-channel HFB-based ADC, which are characterized by special relations between the numerators and denominators of the transfer functions of the analog filters.

With implementation cost in mind, we have decided to choose standard analog filters with low-complexity. We could then tolerate high-order synthesis filters to ensure QPR. However, we still use IIR structures for the synthesis filters so as to lower the number of taps, compared to FIR filters. Obviously, we are aware of the issue of stability and take this into account in the optimization described in the following part.

\section{B. Optimization}

The aim of the optimization is to adjust the synthesis filters so as to approach QPR, as specified by a maximum distortion, $\mathrm{G}_{0} \mathrm{dBmax}$ and a maximum of aliasing rejection, $\mathrm{G}_{1} \mathrm{dBmax}$. Thus we are looking for a way to obtain a transfer function $\mathrm{G}_{0}$ close to 1 and to minimize the aliasing function $G_{1}$. These two objectives are integrated into the single criterion

$$
J\left(F_{0}, F_{1}\right)=\left(\left|G_{0}(j \omega)\right|-1\right)^{2}+\beta\left|G_{1}(j \omega)\right|^{2}
$$

where $\beta$ is a parameter that tunes the relative weight of the two terms. The most stringent requirement being aliasing rejection, the largest weight is given to this term. In the case of guard band, the criterion is obviously only applied on the band of interest.

It has yet be reported [10] that the mixed criterion above suffers of local minima which turns the filter synthesis into a difficult, but key, task. In order to find possible (optimum) synthesis filters $F_{0}$ opt and $F_{1}$ opt, we have developed a heuristic approach that rests on the application of two minimizations strategies: a direct simplex search method that minimizes the average energy of the criterion with fast convergence, complemented by a minimax procedure whose particular goal is to lower the local maxima of the criterion especially on the edges of the band [10]. The algorithm also includes a perturbation strategy to avoid local minima. The overall algorithm is depicted in Fig. 2 and described below.

The algorithm focuses on the specification of maximum of aliasing rejection, $\mathrm{G}_{1} \mathrm{dBmax}$. It ends when the target is reached. It optimizes the coefficients of the numerators and denominators of the IIR digital filters.

We use both Matlab functions: fminsearch and fminimax. fminsearch is a direct search method which is based on the Nelder-Mead method. The corresponding algorithm will find the minimum of a function of $\mathrm{N}$ variables. The function fminimax minimizes the worst-case (largest) value of a set of multivariable functions. This is generally referred to as the minimax problem. Both functions start at an initial estimate and may only give local solutions.

Thus, we have to initialize the synthesis filters. To fasten the process of optimization, we choose the synthesis filters optimized for a particular case of digital filter bank, $\mathrm{F}_{0} \mathrm{DT}$ and $F_{1}$ DT. To achieve this, we identify and use the discretetime analog filters that correspond to the actual analog $\mathrm{H}_{0}$ and $\mathrm{H}_{1}$, the continuous-time filters. As we consider IIR filters, we have to stabilize the solutions given by the functions.

As the functions may only give local solutions, a potential issue is to stall in a local minimum, before having reached the target. The procedure tries to escape from local minima by testing small deviations of the coefficients and by increasing the filters orders. We first have to detect a possible local minimum. If two successive results of aliasing rejections have approximately the same value, we propose two ways to resolve it. On the one hand, we add a little deviation that 
should be carefully chosen, to get out of the local minimum. On the other hand, we increase the order of the IIR filters, and we compare the two solutions.

The algorithm is stopped when the targeted performance is reached. We could go further but we prefer to keep the solutions where the IIR filters have the smallest number of taps. Indeed, there is a trade-off between the complexity of the digital filters and the performances.

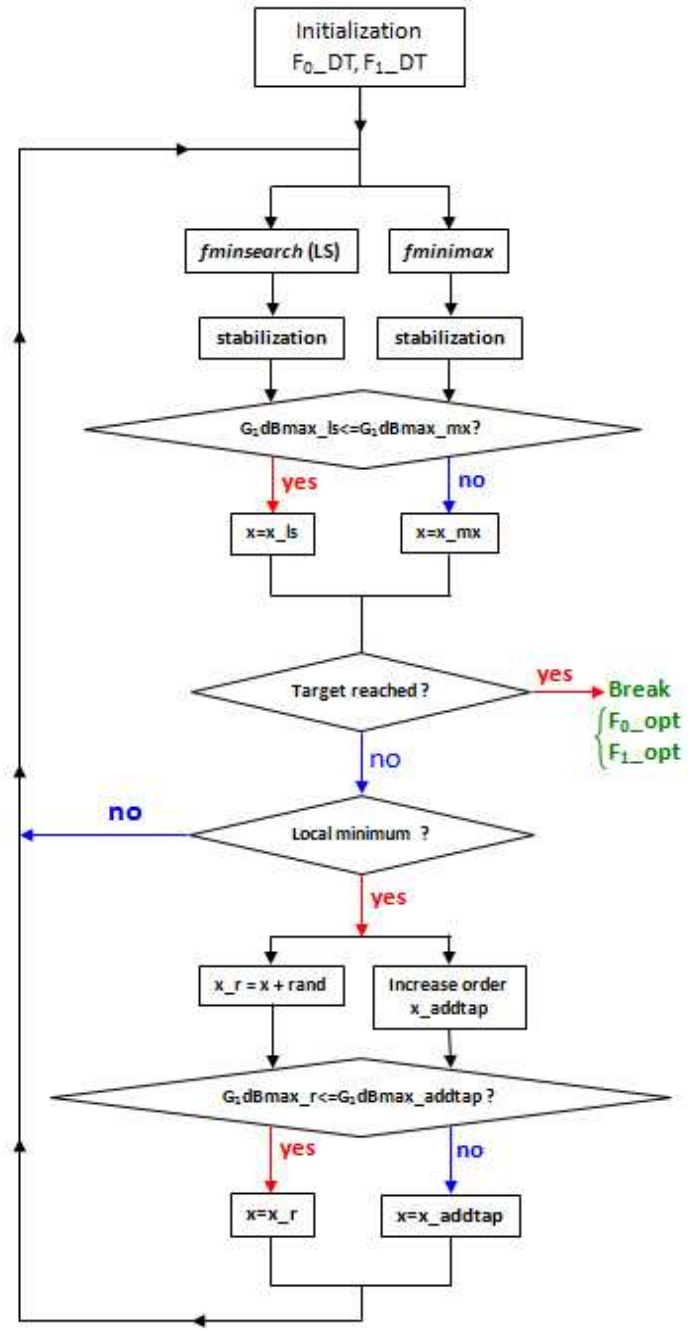

Figure 2. Optimization algorithm

The following table gives an example of performances obtained by simulation when the two analysis filters are chosen as analog $3^{\text {rd }}$-order Butterworth filters.

TABLE I. PERFORMANCES WITH THEORETICAL ANALOG FILTERS

\begin{tabular}{|c|c|c|}
\hline \multicolumn{3}{|c|}{ Performances } \\
\hline$G_{\boldsymbol{0}} \boldsymbol{d B m a x}(\boldsymbol{d B})$ & $\boldsymbol{G}_{\boldsymbol{l}} \boldsymbol{d B m a x}(\boldsymbol{d B})$ & Number of taps* \\
\hline 0.03 & -72.22 & 12 \\
\hline \multicolumn{3}{|c|}{$*$ same for numerator and denominator, and for both synthesis filters }
\end{tabular}

All our tests show that the procedure reaches the targeted performances and gives very interesting results in the case of perfectly known analog filters.

However, as we know, these analysis filters are subject to realization and analog errors and thus deviate from the theoretical filters. It is thus important to examine the influences of such mismatches on the performances.

\section{Realization and analog errors}

Once the synthesis filters have been optimized for ideal analog filters, we introduce errors on the analog filters and examine the performances. More precisely, we more or less change the coefficients of the ideal transfer functions. We choose the example from Table 1 as reference.

\section{TABLE II. PERFORMANCES WITH ACTUAL ANALOG FILTERS}

\begin{tabular}{|c|c|c|}
\hline \multirow{2}{*}{$\begin{array}{c}\text { Analog errors } \\
(\mathbf{\%})\end{array}$} & \multicolumn{2}{|c|}{ Performances } \\
\cline { 2 - 3 } & $\boldsymbol{G}_{\boldsymbol{0}} \boldsymbol{d B m a x}(\boldsymbol{d B})$ & $\boldsymbol{G}_{\boldsymbol{1}} \boldsymbol{d B m a x}(\boldsymbol{d B})$ \\
\hline 10 & 0.99 & -15.86 \\
\hline 1 & 0.09 & -40.39 \\
\hline 0.1 & 0.03 & -56.79 \\
\hline 0.01 & 0.03 & -71.63 \\
\hline
\end{tabular}

We conclude from this table that this architecture is very sensitive to realization and analog errors. This problem has already been reported in [11].

As the actual analog filters could be different from the theoretical filters and since we do not know exactly their transfer functions, the synthesis filters that correspond to the theoretical case are not adapted and the performances are degraded. If we could precisely measure or calibrate the analog filters, it would be easy to optimize the synthesis filters.

\section{IDENTIFICATION}

\section{A. Discussion}

The ideal solution would be to be able to measure the actual analog filters with infinite precision and then to calculate the synthesis filters. However this is not practical because it is difficult to have good precision at high frequencies.

No measure could be done in the analog domain but it is still possible in the digital domain. If we dispose of a known test input signal $x(t)$ with Fourier transform $X(f)$, then we can have access to the outputs of the ADCs, noted $\bar{s}_{0}$ and $\bar{s}_{1}$, given that we bypass the digital filters, cf. Fig. 1. These measures correspond to the outputs of the analog filters, $s_{0}$ and $s_{1}$, with local aliasing, because of undersampling. We thus have the following relationship:

$$
\bar{S}_{k}(f)=S_{k}(f)+S_{k}{ }^{*}(F s-f)
$$

where $F_{S}$ is the sampling rate of each $\mathrm{ADC}$, and

$$
S_{k}(f)=X(f) \times H_{k}(f)
$$

for $\mathrm{k}=0$ or 1 . 
Assuming that we know the input signal and that the measurements have infinite precision, we could then identify the analog filters very well. For this, we use an optimization that operates on the coefficients $a_{k}$ and $b_{k}$ the numerator and denominator of both identified analog filters. We start from the theoretical filters and the aim of the optimization is that the aliased outputs of the identified analog filters match the measured ones:

$$
\inf _{a_{k}, b_{k}}\left|\bar{S}_{k}(f)-\left(X(f) \times H_{k}(f)+X^{*}(F s-f) \times H_{k}{ }^{*}(F s-f)\right)\right|^{2}
$$

This is implemented, again, using the fminsearch function.

In practice, we may not dispose of a pilot signal, but rather we might know that the input signal has the characteristics of a white noise (i.e. decorrelation and flat spectrum). In such a case, the identification criterion can be written with respect to the power spectrum and becomes

$$
\left.\underset{a_{k}, b_{k}}{\inf }|| \bar{S}_{k}(f)\right|^{2}-\left.\left(\left|H_{k}(f)\right|^{2}+\left|H_{k}(F s-f)\right|^{2}\right)\right|^{2}
$$

Using this approach and 128 frequency measurements, the criterion is as low as $10^{-29}$, which indicates that we obtain a perfect identification. Then, from these identified filters, we optimize the synthesis filters and obtain excellent performances, since the estimated filters are close enough to the real analog filters, even if there are analog errors.

Unfortunately, the measurements are not perfect but corrupted by some measurement errors. Indeed, these errors include both modeling errors (e.g. the assumption of a flat spectrum) and errors related to the limited integration time. Therefore, the identification is operated from imperfect measurements. Since the synthesis filters are optimized from these identified analog filters, they will not be well-adapted to the actual analog filters. Simulations results show that the performances are affected and very dependent on the precision.

\section{B. Results}

Table III gives the performances of the architecture when the analog filters are imperfectly known: errors are introduced on the values of the filters coefficients, and the identification procedure is used so as to estimate the actual filters. In this first case, the measurements of the spectrum are supposed free of errors. For different levels of errors, the identification procedure yields excellent results and performances follow.

TABLE III. PERFORMANCES WITH ACTUAL ANALOG FILTERS

\begin{tabular}{|c|c|c|c|}
\hline \multirow{2}{*}{$\begin{array}{c}\text { Analog errors } \\
(\%)\end{array}$} & \multicolumn{3}{|c|}{ Performances } \\
\cline { 2 - 4 } & $\begin{array}{c}\text { Identification } \\
\text { precision }\end{array}$ & $\boldsymbol{G}_{0} \boldsymbol{d B m a x}(\boldsymbol{d B})$ & $\boldsymbol{G}_{1}$ dBmax (dB) \\
\hline 0 & $2.65 \mathrm{E}-29$ & 0.03 & -72.22 \\
\hline 0.01 & $2.64 \mathrm{E}-29$ & 0.03 & -72.07 \\
\hline 0.1 & $2.61 \mathrm{E}-29$ & 0.04 & -70.29 \\
\hline 1 & $2.90 \mathrm{E}-29$ & 0.04 & -71.45 \\
\hline 10 & $3.27 \mathrm{E}-29$ & 0.04 & -70.70 \\
\hline
\end{tabular}

Next we examine the impact of measurement errors on the spectra computed at the output of ADCs and the performances that follow. We see that the performances are severely degraded, due to the imperfect identification. Acceptable performances of the whole design require measurements with more than $60 \mathrm{~dB}$ of SNR.

TABLE IV. PERFORMANCES WITH MEASUREMENT ERRORS

\begin{tabular}{|c|c|c|c|}
\hline \multirow{2}{*}{ SNR (dB) } & \multicolumn{3}{|c|}{ Performances } \\
\cline { 2 - 4 } & $\begin{array}{c}\text { Identification } \\
\text { precision }\end{array}$ & $\boldsymbol{G}_{0} \boldsymbol{d B m a x}(\boldsymbol{d B})$ & $\boldsymbol{G}_{1} \boldsymbol{d B m a x}(\boldsymbol{d B})$ \\
\hline$\infty$ & $2.65 \mathrm{E}-29$ & 0.03 & -72.22 \\
\hline 80 & $5.32 \mathrm{E}-06$ & 0.04 & -69.10 \\
\hline 60 & $6.45 \mathrm{E}-04$ & 0.04 & -54.70 \\
\hline 40 & 0.05 & 0.06 & -39.93 \\
\hline 20 & 5.64 & 0.46 & -17.43 \\
\hline
\end{tabular}

\section{CONCLUSION}

In this paper we have presented an approach to build up an HFB-ADC based on analog filters such as Butterworth or Elliptic filters for the analog analysis bank and on digital IIR filters for the synthesis bank. We have described an optimization scheme involving both direct search and minimax approaches to compute the IIR filters. The identification of the analog analysis filters was also addressed so as to overcome analog imperfections and performances degradation. The results highlight that the proposed methods give very interesting results both for the optimization of the synthesis bank and for calibration, despite that identification appears very sensitive to measurement errors. In order to keep this issue into account, we intend to use a pilot signal, rather than the semi-blind approach used here.

\section{REFERENCES}

[1] W. Black, D. Hodges, "Time interleaved converter arrays," IEEE Journal of Solid-State Circuits, vol. 15, pp. 1022-1029,1980.

[2] I. Galton, H. Jensen, "Oversampling parallel delta-sigma modulator A/D conversion," IEEE trans on Circuits and Systems II: , vol. 43, pp. 801-810, 1996.

[3] S.R. Velasquez, "Hybrid filter banks for analog/digital conversion," $\mathrm{PhD}$ thesis, MIT, 1997.

[4] P. Lowenborg, H. Johansson, L. Wanhammar, "A class of two-channel approximately perfect reconstruction hybrid analog/digital filter banks," Proceedings. ISCAS 2000, pp. 579-582, 2000.

[5] D. Asemani, J. Oksman, "Influences of oversampling and analog imperfections on Hybrid Filter Bank AD Converters", Symposium MWSCAS '06, pp. 123-126, 2006.

[6] S.R. Velazquez, T.Q. Nguyen, S.R. Broadstone, "Design of hybrid filter banks for analog/digital conversion," IEEE trans on Signal Processing, 46, $\mathrm{n}^{\circ} .4$, pp.: 956-967, 1998.

[7] P. Lowenborg, H. Johansson, L. Wanhammar, "A design procedure for 2-channel mixed analog and digital banks for $\mathrm{AD}$ conversion using minimax optimization", Proc ICECS'99, vol. 3, pp. 1189-1192, 1999.

[8] P. Lowenborg, H. Johansson, L. Wanhammar, "A class of two-channel hybrid analog digital filter banks”, Proc ICECS'99, vol. 1, pp. 14-17, 1999.

[9] Liu Zhiyu, Lin Maoliu, "Design of two-channel hybrid analog/digital banks satisfying near-perfect reconstruction," Proc ICSP '04, vol. 1, pp. 407-410, 2004.

[10] R. Koilpillai et P. Vaidyanathan, "Cosine-modulated FIR filter banks satisfying perfect reconstruction," Signal Processing, IEEE Transactions on, vol. 40, $\mathrm{n}^{\circ}$. 4, p. 770-783, 1992

[11] T. Petrescu, J. Oksman, "Sensitivity of hybrid filter banks AD converters to analog realization errors and finite word length", Proc ICASSP'06, pp. 361-364, 2000 